\title{
Associação do estado nutricional com aptidão física relacionada à saúde em adultos com deficiência intelectual
}

CDD. 20.ed. 613.7

796.019

http://dx.doi.org/10.1590/1807-55092015000400543

\author{
Gabriel Renaldo de SOUSA* \\ Marília Garcia PINTO** \\ J efferson Roberto SEEBER ${ }^{* * *}$ \\ Diego Augusto Santos SILVA****
}

*Centro de Ciências da Saúde, Universidade Federal de Santa Catarina.

${ }^{* *}$ Centro de Desportos, Universidade Federal de Santa Catarina.

${ }^{* * *}$ Centro de Educação Física, Fundação Catarinense de Educação Especial.

A obesidade é um problema da saúde pública que tem reflexo direto nos níveis de aptidão física relacionada à saúde, principalmente em pessoas com deficiência intelectual (DI) por uma série de limitações cognitivas e sociais devido à deficiência. 0 objetivo do estudo é identificar a associação do estado nutricional com os níveis de aptidão física relacionado à saúde em pessoas com DI. A pesquisa foi realizada em uma instituição com 31 homens adultos, tendo a avaliação do estado nutricional sido realizada por meio do IMC, e os testes de aptidão física relacionado à saúde com uso da Bateria de Brockport adaptada (avaliação da adiposidade, teste de flexibilidade, força/resistência muscular e capacidade aeróbia). Utilizou-se a análise de covariância para comparar os grupos eutróficos e o excesso de peso em relação aos componentes de aptidão física relacionados à saúde e o teste exato de Fisher para verificar associação entre as variáveis. A maioria dos adultos não atingiu os critérios mínimos para a saúde. Adultos com excesso de peso (IMC) apresentaram maiores valores de percentual de gordura corporal e menores niveis de força/resistência muscular do que os eutróficos. Os indícios da associação entre excesso de peso com altos níveis de percentual de gordura e baixos níveis de força/resistência muscular alerta para a necessidade de programas específicos para população com DI.

Palavras-chave: Deficiência intelectual; Obesidade; Índice de massa corporal; Adulto; Saúde da pessoa com deficiência.

\section{Introdução}

Pessoas com Deficiência Intelectual (DI) são caracterizadas por alguma limitação significativa, tanto no funcionamento intelectual, como também no comportamento adaptativo, expresso em habilidades conceituais, sociais e práticas ${ }^{1}$. Pesquisas ao redor do mundo demonstraram que os baixos níveis de atividade física e o uso de medicamentos (antidepressivos, antipsicóticos e antiepiléticos), são fatores que elevam ainda mais o número de obesos da população com deficiência ${ }^{2-3}$.

A prevalência de obesidade aumenta em todas as regiôes do mundo tanto em países desenvolvidos, como em países em desenvolvimento, tornando-se o maior problema de saúde pública do século $\mathrm{XXI}^{4-5}$. $O$ problema se torna ainda mais grave em pessoas com DI nos quais a prevalência de sobrepeso e obesidade vai de $30 \%$ a $60 \%$ nos Estados Unidos ${ }^{6}$, $34,5 \%$ em países da Europa ${ }^{7}$, cerca de $25 \%$ em países asiáticos ${ }^{8}$, e de $25 \%$ a $60 \%$ no Brasil ${ }^{9-10}$. Essa elevada prevalência de excesso de peso preocupa os órgãos de saúde pública, pois o excesso de gordura ocasiona diversos efeitos negativos à saúde como, o aumento na incidência de doenças respiratórias, distúrbios reprodutivos femininos, osteoartrite, alguns tipos de câncer e, principalmente, aumento do número de doenças crônicas não transmissíveis (diabetes mellitus tipo II, hipertensão arterial sistêmica, doenças coronarianas e dislipidemia) $)^{11-12}$.

Pesquisadores demonstraram que a obesidade está ligada a baixos níveis de aptidão física relacionada à saúde, na população em geral ${ }^{13} \mathrm{e}$ em pessoas com $\mathrm{DI}^{14}$. Frey e CHOw ${ }^{15}$ realizaram pesquisa em Hong 
Kong, avaliando a relação entre índice de massa corporal e aptidão física em 444 jovens com DI e demonstraram que a obesidade afetou negativamente a força/resistência muscular e a capacidade aeróbica. A pesquisa de Salaun e Berthouze-Aranda ${ }^{16}$, na França, que avaliou aptidão física e índice de massa corporal em 87 jovens, encontrou que a obesidade tinha forte relaçáo com níveis baixos de flexibilidade, capacidade aeróbica e de força/resistência muscular.

No Brasil, os estudos que existem sobre a aptidão física relacionada à saúde em pessoas com $\mathrm{DI}^{14,17-18}$ e sobre o estado nutricional desta população ${ }^{9-10,19}$, não caracterizaram de forma clara se avaliaram somente pessoas com essa deficiência. Além disso, tais estudos incluíram, na amostra, pessoas com outras

\section{Método}

\section{Sujeitos da pesquisa}

O estudo foi realizado em uma instituição de educação especial, pública, no município de São José, em Santa Catarina/Brasil que atende pessoas com todos os tipos de deficiência, a qual é responsável por propor políticas, metodologias e pesquisa para todos os indivíduos com deficiência nesse estado brasileiro. A instituiçẫo possuía 113 educandos adultos. Deste quantitativo, 65 apresentavam DI leve à moderada. Todos os 65 adultos foram convidados a participarem da pesquisa. Sete pais nẫo consentiram que o filho e/ou responsável participassem da pesquisa. Desta maneira, 58 adultos estavam elegíveis para participar da pesquisa. Destes 58 adultos, oito foram excluídos porque apresentavam síndrome de Down e 10 porque apresentavam transtorno de espectro autista. Além destes, optou-se por excluir as mulheres $(\mathrm{n}=9)$ porque o quantitativo era baixo, o que limitava o poder das análises estatísticas. Assim, a amostra final foi formada por 31 adultos do sexo masculino que apresentavam DI.

\section{Instrumentos e coleta de dados}

A pesquisa utilizou a bateria de teste de Brockport adaptado para avaliar os níveis de aptidão física relacionados à saúde dos adultos. Essa bateria inclui teste de adiposidade corporal, flexibilidade, força/ resistência muscular e resistência aeróbica. A bateria foi desenvolvida para ser utilizada por pessoas com algum tipo de deficiência, especialmente, aquelas com comprometimento visual, DI e limitaçóes síndromes ou com outros transtornos que, por sua vez, têm características diferentes da DI.

Não foi encontrado, até abril de 2014, nenhum estudo nas bases de dados (Pubmed, Ebsco, Scopus e Google acadêmico), utilizando as palavras chaves "deficiência intelectual" e "aptidão física" e seus descritos em inglês ("intellectual disabilities" e "physical fitness") e espanhol ("discapacidad intelectual" e "aptitud física"), que buscou associar o estado nutricional com componentes da aptidão física relacionada em adultos com DI no Brasil, o que carece de maior investigação. Esse estudo tem como objetivo verificar a associação do estado nutricional com os níveis de aptidáo física relacionada à saúde em adultos com DI.

ortopédicas ${ }^{20}$ e por ser de fácil aplicação e específica para essa população foi empregada nessa pesquisa.

Estado nutricional: Para avaliação do estado nutricional, foram utilizados uma balança da marca Welmy ${ }^{\circledR}$ e um estadiômetro da marca Eggshape ${ }^{\circledR}$, para verificar massa corporal e estatura. Utilizou-se a fórmula de índice de massa corporal = (massa corporal $/$ estatura ${ }^{2}$ ), tendo como referência os valores propostos pela Organização Mundial de Saúde ${ }^{4}$, e aqueles que apresentaram Índice de Massa Corporal (IMC) igual ou menor que $18,4 \mathrm{~kg} / \mathrm{m}^{2}$ foram considerados como baixo peso; entre $18,5 \mathrm{~kg} / \mathrm{m}^{2}$ e $24,9 \mathrm{~kg} / \mathrm{m}^{2}$, peso nor$\mathrm{mal}$; entre $25 \mathrm{~kg} / \mathrm{m}^{2}$ e $29,9 \mathrm{~kg} / \mathrm{m}^{2}$, excesso de peso; e acima $30 \mathrm{~kg} / \mathrm{m}^{2}$, obesos. Tal variável foi dicotomizada em indivíduos eutróficos (indivíduos classificados como peso normal e baixo peso) e excesso de peso (indivíduos classificados com excesso de peso e obesos).

Adiposidade corporal: Para obter o percentual de gordura, foram mensuradas as dobras cutâneas com adipômetro da marca Mitutoyo ${ }^{\circ}$, com precisão de $0,01 \mathrm{~mm}$, e obteve-se a densidade corporal através da fórmula: Densidade $=1,10726863-0,00081201$ (subescapular + tríceps + supra ilíaca + panturrilha medial $)+0,00000212$ (subescapular + tríceps + supra ilíaca + panturrilha medial)2-0,00041761 (idade em anos), proposto por Petroski e Pires Neto ${ }^{21}$. Para calcular o percentual de gordura, foi utilizada a fórmula de Siri: percentual de gordura $=(495 /$ Densidade corporal) - 450. Estabeleceu-se como critério: peso normal quando o percentual de gordura era igual ou inferior a $15 \%$ e, acima desse valor, o sujeito era classificado com excesso de adiposidade corporal ${ }^{20}$. 
Flexibilidade: foi utilizado o teste de flexibilidade com auxílio do Banco de Wells no qual o sujeito, sem os sapatos e sentado em frente ao aparelho, deveria estender os braços acima da escala de medição e empurrar o aparelho de medição, mantendo-o por, pelo menos, um segundo. O movimento foi realizado três vezes e o melhor valor obtido foi computado na pesquisa. Utilizaram-se os pontos de corte propostos por NIEMAN ${ }^{22}$ no qual os sujeitos foram classificados entre condição de risco e baixa aptidão, como "ruim" (entre 15 e 19 anos abaixo de 29 cm; entre 20 a 29 anos abaixo de $30 \mathrm{~cm}$; entre 30 a 39 anos abaixo de 28 segundos; entre 40 a 49 anos abaixo de $20 \mathrm{~cm}$ ) e entre faixa recomendável e condição atlética como "boa", conforme a faixa etária dos sujeitos.

Força/resistência muscular: utilizou-se o teste de pendurar-se com cúbitos flexionados, em que o sujeito da pesquisa ficou suspenso em uma barra com os braços flexionados o maior tempo possível. $\mathrm{O}$ teste era encerrado quando o participante alcançasse a marca de 75 segundos. Foram utilizados os pontos de corte propostos por WinNICK e SHORT ${ }^{20}$ em que é considerado com baixa aptidão os sujeitos que resistem nessa posição por até 15 segundos. Acima desse valor, o adulto é considerado com boa aptidão.

Capacidade aeróbia: Para mensurar a capacidade aeróbia, foi utilizado o teste de campo incremental de vai e vem de Luc-Leger ${ }^{23}$, em que os indivíduos corriam em um ritmo cadenciado pelo áudio, especialmente gravado para este fim, cobrindo um espaço de 20 metros, delimitado entre duas linhas paralelas. A caixa de som emitia bips, em intervalos específicos para cada estágio, sendo que a cada bip, o avaliado deveria cruzar com um dos pés uma das duas linhas paralelas.

Para cálculo da capacidade aeróbia foram utilizadas as recomendaçóes propostas por LEGER e LAMBERT ${ }^{23}$ em que $\mathrm{VO}_{2 \text { máx }}\left(\mathrm{ml} \cdot \mathrm{kg}^{-1} \cdot \mathrm{min}^{-1}\right)=-24,4+6,0^{*}$ y. Sendo

\section{Resultados}

Na TABELA 1, encontram-se os valores médios e o desvio padrão das variáveis coletadas no presente estudo.

$\mathrm{Na}$ TABELA 2, observa-se que mais da metade $(58,1 \%)$ dos sujeitos da pesquisa estavam com excesso de peso, bem como, $83,8 \%$ dos pesquisados, que y é a velocidade dada pela fórmula: Velocidade Atingida $=8+(0,5 \times \mathrm{p})$. O p é o patamar atingido no teste. $\mathrm{OVO}_{2 \text { máx }}$ foi classificado conforme CoOpER ${ }^{24}$, em que as categorias muito fraco e fraco foram agrupadas em "ruim" (abaixo de 38,4 ml. $\mathrm{kg}^{-1}$. min. ${ }^{-1}$ para aqueles com menos de 29 anos; abaixo de $35,5 \mathrm{ml} . \mathrm{kg}^{-1}$. min. ${ }^{-1}$, para aqueles com 30 a 39 anos; menos de 33,6 ml. $\mathrm{kg}^{-1}$. min. ${ }^{-1}$ para aqueles de 40 a 49 anos). Os que atingiram os níveis de capacidade aeróbia regular, boa, excelente e superior, foram categorizados como "boa".

A pesquisa foi aprovada pelo Comitê de Ética e Pesquisa com Seres Humanos da Universidade do Estado de Santa Catarina sob Protocolo CAAE n. 05703712.0.0000.0118.

\section{Análise estatística}

Inicialmente, a normalidade dos dados foi testada por meio de histogramas e do teste de Shapiro-Wilk, com os dados apresentando distribuição normal. Os dados foram analisados por meio da estatística descritiva utilizando valores de média, desvio padrão, erro padrão, frequências absolutas e relativas. Foi verificada a interação entre o estado nutricional com as variáveis de aptidão física. Como não houve interação entre as variáveis, usou-se a análise de covariância (ANCOVA), tendo como covariável, a idade para comparação dos grupos (eutróficos vs. excesso de peso), em relação às variáveis de aptidão física, pois a idade é um fator que interfere no desempenho de testes físicos.

Para verificar a associação entre o estado nutricional com as variáveis de aptidão física, empregou-se o teste Exato de Fisher, pois algumas células apresentavam frequência menor do que cinco. Em todas as análises adotou-se nível de significância de $5 \%(\mathrm{p}<0,05)$. Para análise dos dados, utilizou-se o pacote estatístico SPSS, versão 19.0.

apresentaram elevado percentual de gordura corporal. A maioria dos sujeitos, 74,2\%, obteve nível ruim na medida da flexibilidade e também, nas medidas de força/resistência muscular $(58,1 \%)$ e aptidão aeróbia $(87,0 \%)$. 
TABELA 1 - Valores médios e desvio padrão das variáveis antropométricas e de aptidão física.

M: média;

D.P.: desvio padrão s: segundos;

* densidade corporal.

\begin{tabular}{lc}
\hline Variáveis & M (D.P.) \\
\hline Idade (anos) & $32,4(10,3)$ \\
Massa corporal $(\mathrm{kg})$ & $75,9(14,9)$ \\
Estatura $(\mathrm{cm})$ & $168,4(7,8)$ \\
$\mathrm{IMC}\left(\mathrm{kg} / \mathrm{m}^{2}\right)$ & $26,8(4,9)$ \\
Percentual de gordura $(\%)$ & $23,1(8,1)$ \\
Flexibilidade $(\mathrm{cm})$ & $20,3(8,5)$ \\
Força/resistência muscular $(\mathrm{s})$ & $27,3(22,8)$ \\
Aptidão aeróbia $(\mathrm{ml} / \mathrm{kg} / \mathrm{min})$ & $21,8(7,8)$ \\
\hline
\end{tabular}

TABELA 2 - Frequência absoluta e relativa das variáveis estado nutricional e dos componentes da aptidão física relacionados à saúde.

\begin{tabular}{|c|c|c|}
\hline Variáveis & $\mathbf{n}$ & $\%$ \\
\hline \multicolumn{3}{|l|}{ Estado nutricional } \\
\hline Eutróficos & 13 & 41,9 \\
\hline Excesso de Peso & 18 & 58,1 \\
\hline \multicolumn{3}{|l|}{ Percentual de gordura } \\
\hline Normal & 5 & 16,1 \\
\hline Excesso & 26 & 83,8 \\
\hline \multicolumn{3}{|l|}{ Flexibilidade } \\
\hline Ruim & 23 & 74,2 \\
\hline Bom & 8 & 25,8 \\
\hline \multicolumn{3}{|l|}{ Força/resistência muscular } \\
\hline Ruim & 18 & 58,1 \\
\hline Boa & 13 & 41,9 \\
\hline \multicolumn{3}{|l|}{ Capacidade aeróbia } \\
\hline Ruim & 27 & 87,0 \\
\hline Boa & 4 & 13,0 \\
\hline Total & 31 & 100 \\
\hline
\end{tabular}

Na FIGURA 1, observa-se que os sujeitos com excesso de peso apresentaram maior percentual de gordura corporal $(28,3 \%)$ do que os pares eutróficos $(16,7 \%)$. Por outro lado, os indivíduos eutróficos apresentaram melhor desempenho no teste de força/ resistência muscular do que os sujeitos com excesso de peso $(\mathrm{p}<0,01)$.

Não foi observada nenhuma associação significativa $(p>0,05)$ entre o estado nutricional e a flexibilidade e, entre o estado nutricional e a aptidão aeróbia dos sujeitos. Entretanto, verificou-se associação entre estado nutricional e percentual de gordura, indicando que houve maior proporção de adultos com excesso de peso e com excesso de adiposidade corporal simultaneamente $(\mathrm{p}<0,05)$. Além disso, verificou-se associação entre estado nutricional e força/resistência muscular $(p<0,05)$, indicando que houve maior proporção de indivíduos com excesso de peso e com piores níveis de força/resistência muscular. Por outro lado, houve mais sujeitos com bons níveis de força/resistência muscular classificados como eutróficos (TABELA 3). 


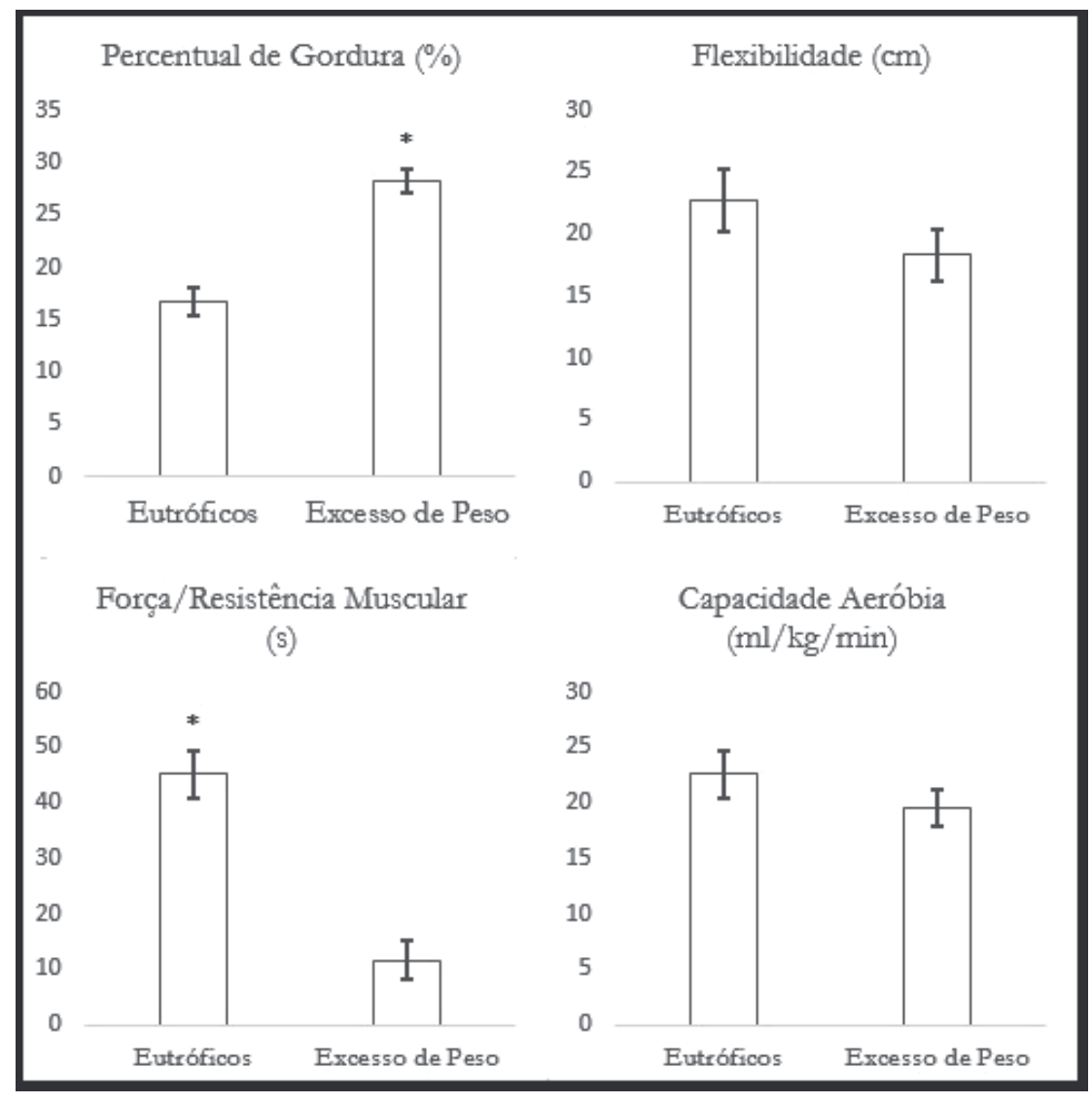

ANCOVA tendo a idade como covariável $(p \leq$ $0,05)$.

FIGURA 1 - Comparação dos valores de média e erro padrão das variáveis de aptidão física relacionadas à saúde de acordo com o estado nutricional.

TABELA 3 - Associação entre estado nutricional e as variáveis de aptidão física relacionada à saúde.

\begin{tabular}{|c|c|c|c|c|}
\hline \multirow{3}{*}{\multicolumn{2}{|c|}{ Variáveis }} & \multicolumn{2}{|c|}{ Grupos } & \multirow{3}{*}{ p-valor } \\
\hline & & Eutróficos & Excesso de peso & \\
\hline & & $\mathrm{n}(\%)$ & $\mathrm{n}(\%)$ & \\
\hline \multicolumn{5}{|c|}{ Percentual de gordura } \\
\hline & Normal & $05(100,0)$ & $00(0,0)$ & $<0,01$ \\
\hline & Excesso & $08(30,8)$ & $18(69,2)$ & \\
\hline \multicolumn{5}{|c|}{ Flexibilidade } \\
\hline & Ruim & $09(39,1)$ & $14(60,9)$ & 0,68 \\
\hline & Boa & $04(50,0)$ & $04(50,0)$ & \\
\hline \multicolumn{5}{|c|}{ Força/resistência muscular } \\
\hline & Ruim & $02(11,1)$ & $16(88,9)$ & $<0,01$ \\
\hline & Bom & $11(84,6)$ & $02(15,4)$ & \\
\hline \multicolumn{5}{|c|}{ Aptidão aeróbia } \\
\hline & Ruim & $11(40,7)$ & $16(59,3)$ & 0,56 \\
\hline & Boa & $02(50,0)$ & $02(50,0)$ & \\
\hline
\end{tabular}




\section{Discussão}

O objetivo principal desta pesquisa foi verificar a associação entre o estado nutricional em relação aos valores de aptidão física relacionada à saúde em pessoas com DI. A amostra continha sujeitos homens, com diagnóstico de DI, ou seja, diferente de todos os outros estudos brasileiros encontrados que incluíram na amostra pessoas com outras síndromes associadas, além da $\mathrm{DI}^{10,14,18-19}$.

Pode-se observar forte relação entre excesso de peso com excesso de gordura corporal investigado por meio das dobras cutâneas. Um estudo com amostra de 12.901 adultos dos Estados Unidos ${ }^{25}$, sem DI, analisou o IMC, a relação cintura-estatura e a circunferência da cintura em relação ao percentual de gordura corporal. Os autores concluíram que o IMC poderia ser usado como indicador de gordura corporal.

$\mathrm{O}$ presente estudo encontrou que pessoas com excesso de peso foram classificadas com piores níveis de força/resistência muscular. Tais resultados convergem com os relatados em Hong Kong com amostra de 31 pessoas jovens com $\mathrm{DI}^{15}$. Além desse estudo de Hong Kong, o presente achado também está de acordo com os reportados por Fernhall e Pitettĩ ${ }^{26}$, que informaram que jovens com DI classificados com excesso de peso, apresentaram níveis de força/resistência muscular inferiores àqueles que não tinham excesso de peso. Tal interpretação dos resultados também pode ser notada durante os testes, pois os sujeitos que tinham sobrepeso, não conseguiam ficar na barra durante muito tempo, provavelmente, devido ao excesso de peso corporal.

Com relação ao estado nutricional, $56,1 \%$ da amostra foi classificada com excesso de peso e/ou obesidade, número que contradiz com a pesquisa de SiLva e SiLva ${ }^{10}$ na qual 34\% dos sujeitos com DI e outras síndromes apresentavam sobrepeso/obesidade. Em outras regiōes do mundo, podem-se encontrar resultados inferiores, como na França em que 34,5\% dos adultos com DI apresentavam excesso de peso e obesidade ${ }^{7}$. Na Coréia do Sul, pesquisa com 890 jovens com DI reportou que a prevalência de excesso de peso e obesidade ficou em torno de $25 \%{ }^{8}$.

Diferenças como essas podem ser explicadas por diversas causas: a) não citaçẫo nos artigos se os investigados tinham somente DI. O que a literatura já mostra são que algumas síndromes, devido a mudanças genéticas, podem trazer efeito no metabolismo, podendo ocasionar ganho maior de peso corporal a essas pessoas ${ }^{3,8,27}$; b) a região em que as pessoas foram investigadas é outro fator que influencia nos resultados do $\mathrm{IMC}^{28}$, pois nos países em desenvolvimento, observa-se número superior de indivíduos com IMC baixo devido ao menor acesso a a limentos e deslocamentos ativos diários ${ }^{29} \mathrm{e}$; c) o baixo nível de atividade física ${ }^{30}$, combinada com a ingestão excessiva de alimentos, pode ocasionar problemas, como maior risco de hipertensão arterial, doenças cardiovasculares, respiratórias, distúrbios reprodutivos femininos, diabetes tipo II, dislipidemias, osteoartrite e alguns tipos de câncer ${ }^{31}$.

Não houve associação entre o estado nutricional e os componentes de flexibilidade e de aptidão aeróbia. Esse resultado difere do estudo de SALAUn e BERTHOU$\mathrm{ZEN}^{16}$, que encontraram associação entre obesidade, flexibilidade $\mathrm{VOO}_{2 \text { max. }}$ em jovens com DI. Os autores encontraram que jovens com obesidade tinham piores resultados em tais componentes da aptidáo física do que os eutróficos ${ }^{17}$. Na população em geral, a obesidade, além de ocasionar diversos outras problemas de saúde, diminui os níveis de aptidão física ${ }^{32}$.

Uma das limitaçôes do estudo foi o número de sujeitos encontrados para participar da pesquisa. Esse quantitativo de pessoas impede a generalização dos resultados para toda a populaçáo de adultos com DI do Brasil. Dessa forma, é importante que mais pesquisas com essa população sejam realizadas em outros estados do país. A amostra, também, só contou com participantes homens, e conforme as pesquisas demonstraram, há evidências fortes de que mulheres também apresentam alta tendência ao excesso de peso $^{7,28,31}$. Por ser uma pesquisa de corte transversal, também não se pode dizer que o excesso de peso leva a níveis baixos de aptidão física relacionados à saúde ou níveis baixos de aptidáo física relacionados à saúde refletem no excesso de peso, necessitando estudos longitudinais para esta população.

Com relação aos instrumentos utilizados, cabe ressaltar que pessoas com excesso de peso apresentam maior dificuldade em realizar testes de aptidáo física. Em futuras pesquisas, pode-se pensar, por exemplo, em analisar o percentual de gordura por meio de métodos indiretos de avaliaçáo da composição corporal. Foi verificado, nesta pesquisa, que em uma amostra de homens adultos com DI, o excesso de peso investigado por meio do IMC refletiu em maior percentual de gordura e em menor desempenho no teste de força/resistência muscular.

Tanto os profissionais de Educação Física como os das demais áreas da saúde, necessitam trabalhar em conjunto para que o número de pessoas com obesidade, nesta populaçáo, possa diminuir e assim, trazer benefícios à saúde e melhor independência nesta população. 


\section{Abstract}

Association between nutritional status and of health-related fitness in adults with intellectual disabilities

Obesity is a public health problem that has a direct impact on levels of physical fitness and health, especially in people with intellectual disabilities (ID) for a series of cognitive and social limitations due to disability. The objective of the study is to identify the association between nutritional status and levels of healthrelated fitness in people with ID. The research was conducted in an institution with 31 adult men that the assessment of nutritional status was performed using BMI, and physical fitness tests related to health with use of adapted Brockport battery (assessment of adiposity, flexibility test, muscular strength / endurance and aerobic capacity). If you used the covariance analysis to compare groups eutrophic and overweight in relation to the components of physical fitness and health and the Fisher exact test to verify the association between variables. Most adults did not meet the minimum criteria for health. Adults who are overweight (BMI) had higher percentage values of body fat and lower levels of muscular strength / endurance than eutrophic. The Association of evidence between overweight with high percentage of fat levels and low levels of muscular strength / endurance alert to the need for specific programs for people with ID.

KEY WoRDs: Intellectual disability; Obesity; Body mass index; Adult ; Health of the disabled.

\section{Referências}

1. Schalock RL, Borthwick-Duffy SA, Bradley VJ, et al. Intellectual disability: definition, classification, and systems of supports. 11th ed. Washington: AAIDD; 2010.

2. Winter CF, Bastiaanse LP, Hilgenkamp TI, Evenhuis HM, Echteld MA. Overweight and obesity in older people with intellectual disability. Res Dev Disabil. 2012;33:398-405.

3. Melville CA, Hamilton S, Hankey CR, Miller S, Boyle S. The prevalence and determinants of obesity in adults with intellectual disabilities. Obes Rev. 2007;8:223-30.

4. WHO. World Health Organization. Diet, nutrition and the prevention of chronic diseases. Geneva: WHO; 2002. WHO Technical Report Series.

5. Gazizova D, Puri BK, Singh I, Dhaliwal R. The overweight: obesity and plasma lipids in adults with intellectual disability and mental illness. J Intellect Disabil Res. 2012;56:895-901.

6. Rimmer JH, Yamaki K. Obesity and intellectual disability. Ment Retard Dev Disabil Res Rev. 2006;12:22-7.

7. Begarie J, Maiano C, Leconte P, Ninot G. The prevalence and determinants of overweight and obesity among French youths and adults with intellectual disabilities attending special education schools. Res Dev Disabil. 2013;34:1417-25.

8. Choi E, Park H, Ha Y, Hwang WJ. Prevalence of overweight and obesity in children with intellectual disabilities in Korea. J Appl Res Intellect Disabil. 2012;25:476-83.

9. Raulino AGD, Barros JF. Estudo do comportamento da composição corporal em homems portadores de deficiência mental no Distrito Federal. Rev Bras Ciênc Mov. 2002;10:63-70.

10. Silva R, Silva GP. Características antropométricas e nutricionais de pessoas com deficiência mental. Fit Perform J. 2009;8:130-5.

11. Jakobsen MU, O’Reilly EJ, Heitmann BL, et al. Major types of dietary fat and risk of coronary heart disease: a pooled analysis of 11 cohort studies. Am J Clin Nutr. 2009;89:1425-32.

12. Sarturi JB, Neves J, Peres KG. Obesidade em adultos: estudo de base populacional num município de pequeno porte no sul do Brasil em 2005. Ciênc Saúde Colet. 2010;15:105-13.

13. Teixeira CS, Pereira ÉF. Aptidão física, idade e estado nutricional em militares. Arq Bras Cardiol. 2010;94:438-43.

14. Bacciotti SM. Avaliação da aptidão física relacionada à saúde em indivíduos de 8 a 17 anos com deficiência mental da APAE de Campo Grande-MS [dissertação]. Brasília (DF): Universidade de Brasília; 2009.

15. Frey GC, Chow B. Relationship between BMI, physical fitness, and motor skills in youth with mild intellectual disabilities. Int J Obes. 2006;30:861-7.

16. Salaun L, Berthouze-Aranda SE. Physical fitness and fatness in adolescents with intellectual disabilities. J Appl Res Intellect Disabil 2012;25:231-9. 
17. Barros JF, Pires Neto CS, Barros Neto TL. Estudo comparativo das variáveis neuro-motoras em portadores de deficiência mental. Rev Bras Ciênc Mov. 2000;8:43-8.

18. Mauerberg-de Castro E, Tavares CP, Panhan AC, et al. Educação física adaptada inclusiva: impacto na aptidão física de pessoas com deficiência intelectual. Rev Ciênc Ext. 2013;9:35-61.

19. Silva DL, Santos JAR, Martins CF. Avaliação da composição corporal em adultos com Síndrome de Down. Arq Med. 2006;20:103-10.

20. Winnick JP, Short FX. The Brockport physical fitness training guide. Champaign: Human Kinetics; 1999.

21. Petroski EL, Pires Neto CS. Validação de equaçóes antropométricas para a estimativa da densidade corporal em homens. Rev Bras Ativ Fís Saúde. 1996;1:5-14.

22. Nieman DC. Exercicio físico e saúde. São Paulo: Manole; 1999.

23. Léger LA, Lambert J. A maximal multistage 20-m shuttle run test to predict $\backslash$ dot $\mathrm{VO}_{2 \text { max }}$. Eur J Appl Physiol Occup Physiol. 1982;49:1-12

24. Cooper KH. The aerobics program for total well-being: exercise, diet, emotional balance. New York: Bantam Books; 1982.

25. Flegal KM, Shepherd JA, Looker AC, et al. Comparisons of percentage body fat, body mass index, waist circumference, and waist-stature ratio in adults. Am J Clin Nutr. 2009;89:500-8.

26. Fernhall B, Pitetti KH. Leg strength is related to endurance run performance in children and adolescents with mental retardation. Pediatr Exerc Sci. 2000;12:324-33.

27. Maiano C. Prevalence and risk factors of overweight and obesity among children and adolescents with intellectual disabilities. Obes Rev. 2011;12:189-97.

28. Astell-Burt T, Feng X, Croteau K, Kolt GS. Influence of neighbourhood ethnic density, diet and physical activity on ethnic differences in weight status: a study of 214,807 adults in Australia. Soc Sci Med. 2013;93:70-7.

29. WHO. World Health Organization. Obesity: preventing and managing the global epidemic. Geneva: WHO; 2000.

30. George V, Shacter S, Johnson P. BMI and attitudes and beliefs about physical activity and nutrition of parents of adolescents with intellectual disabilities. J Intellect Disabil Res. 2011;55:1054-63.

31. Casey AF. Measuring body composition in individuals with intellectual disability: a scoping review. J Obes. 2013;2013:6.

32. Farrell SW, Finley CE, Radford NB, Haskell WL. Cardiorespiratory fitness, body mass index, and heart failure mortality in men: Cooper Center Longitudinal Study. Circ Heart Fail. 2013;6:898-905.

\begin{tabular}{r|l} 
ENDEREÇO & \\
Gabriel Sousa & \\
Centro de Desportos & Recebido para publicação: 19/ 12/2014 \\
Universidade Federal de Santa Catarina & 1a. revisão: 21/07/2015 \\
Campus Universitário Trindade & 2a. revisão: 31/08/2015 \\
88010-970 - Florianópolis - SC - BRASIL & Aceito: 21/09/2015 \\
e-mail: gabrieldesousa@gmail.com & \\
&
\end{tabular}

550 • Rev Bras Educ Fís Esporte, (São Paulo) 2015 Out-Dez; 29(4):543-50 